\title{
Mechanical Ventilation Therapy on Covid-19
}

\author{
Arief Kurniawan* \\ Anaesthesiology Department, Medical Faculty \\ Universitas Jenderal Achmad Yani \\ Cimahi, Indonesia \\ *konsultanKIC@gmail.com
}

\begin{abstract}
Covid 19 patient in critical condition characterized by Acute Respiratory Distress Syndrome (ARDS) for which the mainstay of treatment is represented by mechanical ventilation, sepsis and septic shock that require immediate treatment to save his life. This Paper is a literature review of mechanical ventilation therapy on Covid 19 through the search of textbook and accredited journal sites. Currently the pulmonary manifestations of Covid-19 are described as a spectrum with 2 points. The starting point is infection Covid-19 type $L$ which responds to oxygen therapy conventional and requiring Covid-19 type $H$ infection oxygen therapy with higher pressure. As initial therapy, give oxygen with a nasal cannula or face mask, if it is not responding use High Flow Nasal Canule (HFNC). NonInvasive Ventilation (NIV) is considered if there are no signs of immediate need for intubation but must be accompanied by close monitoring with a target $\mathrm{SpO2}$ of not more than $96 \%$. Immediately intubate and give mechanical ventilation if this occurs deterioration during use of HFNC or NIV or not improving within 1 hour. Ventilation mode may use volume or pressure based with the recommended Tidal Volume range is 4-8 ml / kgbw. Use end-expiratory positive pressure (PEEP) limit height in type $H$ while in type $L$ limit with a maximum PEEP of 8-10 cmH2O. The breathing rate is regulated by calculation adequate minute ventilation with a plateau pressure (Pplat) $<30$ cmH2O. If refractory hypoxemia occurs, have pulmonary recruitment, and consider extracorporeal membrane oxygenation (ECMO) therapy. Mechanical ventilation therapy requires initiation, steps, and appropriate ventilator settings according to the severity of Covid-19.
\end{abstract}

Keywords-Covid-19, HFNC, mechanical ventilation, NIV, PEEP, Pplat

\section{INTRODUCTION}

Coronavirus Disease 2019 (COVID-19) is a contagious disease caused by Severe Acute Respiratory Syndrome Coronavirus 2 (SARS-CoV-2). SARS-CoV-2 is a new type of coronavirus that has never been previously identified in humans. There are at least two types of coronaviruses that are known to cause diseases that have severe symptoms such as Middle East Respiratory Syndrome (MERS) and Severe Acute Respiratory Syndrome (SARS). Common signs and symptoms of COVID-19 infection include acute respiratory symptoms such as fever, cough, and shortness of breath. The average incubation period is 5-6 days with the longest incubation period of 14 days. Severe cases of COVID-19 can cause pneumonia, acute respiratory syndrome, kidney failure and even death [1].

\section{Clinical Management OF Covid-19}

Clinical management is a series of activities carried out by medical and health personnel to establish a diagnosis, carry out treatment management and act against COVID-19 patients according to clinical indications. The medical personnel involved as the Doctor in Charge of Services (DCS) are pulmonary specialists, internal medicine doctors, sub-specialist in pulmonary disease, sub-specialist in tropical infectious diseases, pediatricians, pediatric pulmonary sub specialists, and other specialists or other sub-specialist doctors according to medical needs [1].

Clinical management is carried out on COVID-19 patients without symptoms, mild illness, moderate illness, severe illness, critical conditions, and in certain conditions. Immediate oxygen supplementation therapy for patients with severe ARS and patients experiencing respiratory distress, hypoxemia, or shock. Oxygen therapy starts with $5 \mathrm{~L} / \mathrm{min}$ with nasal cannula and titration to achieve the target $\mathrm{SpO} 2 \geq 90 \%$ in children and adults, and $\mathrm{SpO} 2 \geq 92 \%-95 \%$ in pregnant patients [2].

\section{MANAGEMENT OF HYPOXEMIC RESPIRATORY FAILURE AND ARDS}

Hypoxemic respiratory failure may occur in patients with respiratory distress who have failed standard oxygen therapy. Patients may experience increased respiratory work or hypoxemia despite being given oxygen via a facepiece with a reservoir bag (10 to $15 \mathrm{~L} \mathrm{/} \mathrm{min,} \mathrm{minimal} \mathrm{flow} \mathrm{required} \mathrm{to}$ inflate the bag; FiO2 between 0.60 and 0.95). Hypoxemic respiratory failure in ARDS results from improper ventilationperfusion and usually requires mechanical ventilation [2].

High-flow nasal oxygen (HFNO) or non-invasive ventilation (NIV) is given only in certain hypoxemic respiratory failure patients, and these patients should be closely monitored for clinical deterioration. The HFNO system can provide oxygen flows of up to $60 \mathrm{~L} / \mathrm{min}$ and $\mathrm{FiO} 2$ to 1.0; Pediatric circuits generally only reach $15 \mathrm{~L} / \mathrm{min}$, so many children need adult circuits to provide adequate flow. Compared to standard oxygen therapy, HFNO reduces the need 
for intubation. Patients with hypercapnia (exacerbation of obstructive pulmonary disease, cardiogenic pulmonary edema), hemodynamically unstable, multi-organ failure, or decreased consciousness should not use HFNO, although recent data suggest that HFNO may be safe in mild-moderate hypercapnia patients without worsening. Patients with HFNO should be monitored by personnel who are trained and experienced in endotracheal intubation because if the patient experiences sudden deterioration or does not experience improvement (within 1 hour) then intubation is performed immediately. Currently evidence-based guidelines on HFNO do not exist, and reports on HFNO in MERS patients are limited [3].

\section{INTUBATION TIMING}

Patients with NIV should be monitored by trained and experienced personnel to perform endotracheal intubation because if the patient experiences sudden deterioration or does not experience improvement (within 1 hour) then intubation is performed immediately [4]

Endotracheal intubation should be performed by trained and experienced personnel with attention to airborne precautions. Patients with ARDS, especially young children, obese or pregnant, may desaturate rapidly during intubation. Patients were pre-oxygenated before intubation with $100 \%$ Oxygen Fraction (FiO2) for 5 minutes, through a face mask with an air bag, bag-valve mask, HFNO or NIV and then continued with intubation [4].

\section{Mechanical Ventilation SETtings}

Mechanical ventilation uses a low tidal volume $(4-8 \mathrm{ml} / \mathrm{kg}$ predictive body weight, Predicted Body Weight / PBW) and low inspiratory pressure (plateau pressure $<30 \mathrm{cmH} 2 \mathrm{O}$ ). It is highly recommended for ARDS patients and recommended in patients with respiratory failure due to sepsis who do not meet ARDS criteria [4].

Calculate PBW for men $=50+2.3$ [height (inches) -60 ], women $=45.5+2.3$ [height (inches) -60]. Select mechanical ventilation mode. Adjust mechanical ventilation to achieve an initial tidal volume $=8 \mathrm{ml} / \mathrm{kg}$ PBW. Gradually reduce the initial tidal volume by $1 \mathrm{ml} / \mathrm{kg}$ over $\leq 2$ hours until reaching a tidal volume $=6 \mathrm{ml} / \mathrm{kg} \mathrm{PBW}$. Adjust the breath rate to achieve minute ventilation (no more than 35 beats / minute). Adjust the tidal volume and breath rate to reach a target $\mathrm{pH}$ and plateau pressure [4].

Hypercapnia is allowed if the $\mathrm{pH}$ is 7.30-7.45. Mechanical ventilation protocols must be in place. Use of deep sedation to control breath effort and achieve target tidal volume. The prediction of increased mortality in ARDS is more accurate using a high driving pressure (plateau pressure - PEEP) as opposed to a high tidal volume or plateau pressure [5]

\section{INCREASING OXYGEN SATURATION}

In patients with severe ARDS, ventilate them in a prone position> 12 hours per day. Prone position ventilation is highly recommended for adult and pediatric patients with severe ARDS but requires adequate human resources and expertise. Conservative fluid management is used for ARDS patients without tissue hypoperfusion. This is highly recommended because it can shorten the use of a ventilator [6].

Patients with moderate or severe ARDS are advised to use a higher PEEP than a low PEEP. PEEP titration is necessary considering benefits (reduced atelectrauma and increased alveolar recruitment) and risk (excess pressure at the end of inspiration leading to pulmonary parenchymal injury and higher pulmonary vascular resistance). To guide the PEEP titration based on $\mathrm{FiO} 2$ required to maintain $\mathrm{SpO} 2$. Intervention recruitment maneuvers (RMs) are performed periodically with a high CPAP [30-40 $\mathrm{cm} \mathrm{H} 2 \mathrm{O}]$, a progressive increase in PEEP with constant driving pressure, or high driving pressure considering the benefits and risks [6]

In patients with moderate to severe ARDS ( $\mathrm{PaO} 2 / \mathrm{FiO} 2$ $<150)$ it is not recommended to routinely use muscle relaxants. For health facilities that have expertise in Extra Corporal Life Support (ECLS), its use can be considered when receiving referrals for patients with refractory hypoxemia even though they have received lung protective ventilation. There are currently no guidelines that recommend the use of ECLS in patients with ARDS, but there are studies that it may reduce the risk of death. Avoid disconnecting mechanical ventilation from the patient as this can result in loss of PEEP and atelectasis. Use a system of closed suction catheters and endotracheal tube clamps when mechanical ventilation and patient are disconnected (for example, when transfer to portable mechanical ventilation). Treat septic shock whenever exist characterized by hypotension that persists despite fluid resuscitation and requires vasopressors to maintain MAP $\geq 65$ $\mathrm{mmHg}$ and serum lactate levels $>2 \mathrm{mmol} / \mathrm{L}$ [6]

\section{COMPLICATION PREVENTION}

Reducing the length of days used for invasive mechanical ventilation (IMV) with a weaning protocol includes daily assessment of readiness for spontaneous breathing. Apply minimal periodic or continuous sedation, titrate to achieve specific targets (although light sedation is contraindicated) or with daily interruptions from continuous sedation infusion [7].

Reducing the incidence of ventilator-associated pneumonia (VAP) with better oral intubation than nasal intubation in adolescents and adults. Keep the patient in a semi-recumbent position (raise the patient's head to form an angle of 30-45 degrees). Use a closed suctioning system and drain the condensate in the pipe periodically. Each patient uses a new ventilator circuit, changing the circuit if it is dirty or damaged. Change the heat moisture exchanger (HME) if it does not work when it is dirty or every 5-7 days [7].

It is also necessary to take measures to reduce the occurrence of venous thromboembolism, catheter-related bloodstream-related infections, pressure ulcers, stress ulcers, gastrointestinal bleeding, and the occurrence of weakness due to treatment in the ICU [8]. 


\section{CONCLUSION}

Mechanical ventilation therapy is part of a comprehensive therapy for Covid-19 sufferers, which consists of pharmacological and non-pharmacological therapy. Evaluation of the patient's clinical status was carried out to determine ventilator weaning until he was declared cured.

Confirmed case patients with severe / critical symptoms who are admitted to the hospital who have undergone isolation for 10 days since onset plus at least 3 days no longer show symptoms of fever and respiratory problems, are declared complete isolation, and can be transferred to non-isolation ward or be discharged.

\section{REFERENCES}

[1] J. Phua, L. Weng, L. Ling, M. Egi, C.M. Lim, J.V. Divatia and Asian Critical Care Clinical Trials Group, "Intensive care management of coronavirus disease 2019 (COVID-19): challenges and recommendations," The lancet respiratory medicine, vol. 8, no. (5), pp. 506-517, 2020.
[2] ESICM, COVID-19 Ventilation Clinical Practice Guidelines, 2020.

[3] W. Alhazzani, M.H. Møller, Y.M. Arabi, M. Loeb, M.N. Gong, E. Fan and A. Rhodes, "Surviving Sepsis Campaign: guidelines on the management of critically ill adults with Coronavirus Disease 2019 (COVID-19)," Intensive care medicine, vol. 46, no. (5), pp. 854-887, 2020 .

[4] L. Gattinoni, D. Chiumello, P. Caironi, M. Busana, F. Romitti, L. Brazzi and L. Camporota, COVID-19 pneumonia: different respiratory treatments for different phenotypes?. New York: Springer.

[5] L. Gattinoni, S. Coppola, M. Cressoni, M. Busana, S. Rossi and D. Chiumello, "COVID-19 does not lead to a "typical" acute respiratory distress syndrome," American journal of respiratory and critical care medicine, vol. 201, no. (10), pp. 1299-1300, 2020.

[6] L. Camporota and C. Guerin, "How to ventilate in COVID-19," ESICM Webinar, vol. 4, no. (2), 2020.

[7] E. Fan, J.R. Beitler, L. Brochard, C.S. Calfee, N.D. Ferguson, A.S. Slutsky and D. Brodie, "COVID-19-associated acute respiratory distress syndrome: is a different approach to management warranted?" The Lancet Respiratory Medicine, vol. 8, no. (8), pp. 816-821, 2020.

[8] A. Boscolo, L. Spiezia, C. Correale, N. Sella, E. Pesenti, L. Beghetto and P. Navalesi, "Different hypercoagulable profiles in patients with COVID-19 admitted to the internal medicine ward and the intensive care unit," Thrombosis and Haemostasis, vol. 120, no. (10), pp. 1474-1477, 2020 